PUPT-1481

\title{
Free energy decreases along Wilson renormalization group trajectories
}

\author{
Vipul Periwal ${ }^{\dagger}$ \\ Department of Physics \\ Princeton University \\ Princeton, New Jersey 08544-0708
}

The free energy is shown to decrease along Wilson renormalization group trajectories, in a dimension-independent fashion, for $d>2$. The argument assumes the monotonicity of the cutoff function, and positivity of a spectral representation of the two point function. The argument is valid to all orders in perturbation theory.

\footnotetext{
$\dagger$ vipul@puhep1.princeton.edu
} 
The nature of the flow induced by the renormalization group ( $\mathrm{rg}$ ) on the space of couplings in a field theory has been of interest for some time[1,2,3]. Zamolodchikov's $c$-theorem[4] rekindled interest in demonstrating the dissipative character of the renormalization group. He demonstrated that there is a function on the space of renormalizable couplings of a two-dimensional field theory with the following properties:

(i) $\mathrm{d} c=0$ at fixed points,

(ii) the value of $c$ at these fixed points is given by the conformal anomaly, and

(iii) $c$ decreases along rg trajectories, i.e., $\mathrm{d} c(\beta)<0$, where $\beta$ is the vector field associated with $\beta$-functions via $\beta \equiv \beta^{i} \partial_{i}$.

Zamolodchikov's argument used the conventional field theory $\operatorname{rg}[5]$, with $\beta$ satisfying the Callan-Symanzik equations - the couplings that he needed to consider were only those associated with marginal and relevant operators. The $c$ function he found has universal properties, and may therefore be of physical interest even setting aside the question of the dissipative character of the field theory rg.

There is another approach to renormalization due to Wilson[6], where one studies the flows of all couplings in a cut-off theory. This set of flows is not universal. The cut-off is present at all stages since one has irrelevant operators of arbitrarily high dimension in the action. Nevertheless, as can be demonstrated explicitly, the information contained in the field theory $\mathrm{rg}$ can be extracted from the Wilson $\mathrm{rg}[7]$. I shall demonstrate within the Wilson rg that the free energy decreases along Wilson rg trajectories, independent of the dimension of spacetime, for $d>2$. Thus the Wilson $\mathrm{rg}$ is dissipative, for the class of theories that I am able to consider. The argument is completely different in character from Zamolodchikov's. It is unlikely that the free energy will satisfy either (i) or (ii). The decrease of the free energy also does not imply gradient flow, i.e., I do not claim that the Wilson rg beta 'functions' are gradients. I emphasize that the entire framework of the argument is restricted to all orders in perturbation theory. No statements beyond perturbation theory are suggested or implied in any way.

The field-theoretic rg is not trivially related to the Wilson $\mathrm{rg}$ - for some results in this area, see Refs. 8,7. While the intuition for the Zamolodchikov $c$ theorem is usually couched in terms of 'thinning out degrees of freedom', more appropriate to the Wilson framework, the decrease of the free energy along Wilson rg trajectories does not straightforwardly imply the existence of a $c$-function on the sub-manifold of marginal and relevant couplings.

Attempts to generalize Zamolodchikov's argument to dimensions larger than two in simple ways fail because the rotation group is no longer Abelian. For a review of such attempts, and an alternative proof of Zamoldchikov's c-theorem, see Ref. 10. For Wilson rg arguments, see Ref. 12. Cardy[9] suggested that the trace anomaly on a sphere was the 
analogue of the central charge in two dimensions, but the subtractions necessary to define integrals of two-point functions in continuum field theory render it difficult to show (iii).

I give now a general argument for the decrease of the free energy for Wilson rg flows. I will work with Euclidean field theories. The argument is valid for all theories which can be regulated by including a cut-off dependence explicitly in the action. This is not, perhaps, as large a class as one wants, since it amounts to theories that (up to cut-off dependent rescalings of fields) can be regulated by modifying the kinetic term appropriately. It applies to all scalar field theories, and may be extended to theories with fermions for which one can show the validity of the assumption on the spectral measure, see below.

The argument requires the derivation of a new spectral representation - this spectral representation makes no reference to cutoffs or to the renormalization group, and hence may be of independent interest. The derivation is a slight modification of a derivation given in Ref. 10. Lorentz invariance implies that the identity operator can be decomposed as

$$
1=\int_{0}^{\infty} \mathrm{d} \mu^{2} \int_{M} \mathrm{~d}^{d} p \delta\left(p^{2}+\mu^{2}\right) \delta^{(n)}(\hat{p}-p) \theta\left(p_{0}\right),
$$

with $\hat{p}$ the momentum operator and $p_{\alpha}=\left(p_{0}, \mathbf{p}\right)$ its Minkowski eigenvalue. The subscript $M$ on the integral implies integration over Minkowski momenta. For any monotonically increasing positive function, $f$, this can be rewritten as

$$
1=\int_{0}^{\infty} \mathrm{d}\left(\mu^{2} f\left(\mu^{2}\right)\right) \int_{M} \mathrm{~d}^{d} p \delta\left(p^{2} f\left(\left|p^{2}\right|\right)+\mu^{2} f\left(\mu^{2}\right)\right) \delta^{(d)}(\hat{p}-p) \theta\left(p_{0}\right) .
$$

Inserting it into a two-point function (with $x^{d}<0$ ) we have

$$
\langle\phi(x) \phi(0)\rangle=\int \mathrm{d} m^{2} \int_{M} \mathrm{~d}^{d} p \exp (i p x) \delta\left(p^{2} f\left(\left|p^{2}\right|\right)+m^{2}\right) \theta\left(p_{0}\right)\left\langle 0\left|\phi(0) \delta^{(d)}(\hat{p}-p) \phi(0)\right| 0\right\rangle .
$$

The matrix element is independent of $x$, and is just a function of $p^{2}$. The integral over $p$ can be done, giving

$\int_{M} \mathrm{~d}^{d} p \mathrm{e}^{i p x} \delta\left(p^{2} f\left(\left|p^{2}\right|\right)+m^{2}\right) \theta\left(p_{0}\right)=\int \mathrm{d}^{d-1} \mathbf{p} \mathrm{e}^{i \mathbf{p} \cdot \mathbf{x}} \int_{0}^{\infty} \mathrm{d} p_{0} \mathrm{e}^{p_{0} x^{d}} \delta\left(\left(\mathbf{p}^{2}-p_{0}^{2}\right) f\left(\left|\mathbf{p}^{2}-p_{0}^{2}\right|\right)+m^{2}\right)$.

Finally, since $x^{d}<0$ by assumption, the integral over $p_{0}$ with a $\delta$ function can be rewritten as

$$
\int \mathrm{d}^{d-1} \mathbf{p} \exp (i \mathbf{p} \cdot \mathbf{x}) \int_{-\infty}^{\infty} \frac{\mathrm{d} p_{d}}{2 \pi} \exp \left(i p_{d} x^{d}\right) \frac{\theta\left(-x^{d}\right)}{\left(\mathbf{p}^{2}+p_{d}^{2}\right) f\left(\mathbf{p}^{2}+p_{d}^{2}\right)+m^{2}}
$$

Add the term with $x^{d}>0$ to get the propagator in Euclidean space. Thus, putting terms together, one finds the spectral representation

$$
\langle\phi(p) \phi(-p)\rangle=\int_{0}^{\infty} \mathrm{d} m^{2} \rho\left(m^{2}\right) \frac{1}{p^{2} f\left(p^{2}\right)+m^{2}},
$$


with

$$
\rho\left(m^{2}\right)=\left.(2 \pi)^{d-1}\langle 0|\phi(x=0) \delta(\hat{p}-p) \phi(x=0)| 0\rangle\right|_{p^{2} f\left(\left|p^{2}\right|\right)=-m^{2}}
$$

Note that nowhere in this derivation is any use made of the cutoff nature of the theory. Based on this fact, I assume that $\rho$ is positive, since this is a two-point function with the insertion of a positive operator delta function, and the two-point function should be positive even in the cutoff theory.

Let $Z_{0} \equiv \int \prod \mathrm{D} \phi \exp \left(-S_{i n t}(\phi)-\frac{1}{2} \int \phi\left(-\partial^{2}\right) \phi\right)$ be a formal partition function, expressed as a functional integral over some set of fields, $\phi$. A proper definition of $Z_{0}$ requires a cutoff. Let $K(x)$ be a differentiable monotone function such that

$$
K(x)=1 \quad \text { for } x<1, \quad \text { and } \quad K(x) \downarrow 0 \quad \text { for } x \gg 1 .
$$

I assume that $K(x)$ goes to zero faster than any negative power of $x$ for $x$ large. Suppose that

$$
Z_{\epsilon}[J] \equiv \int \frac{\mathrm{D} \phi}{\operatorname{det}^{\frac{1}{2}} K\left(-\partial^{2} / \epsilon^{2}\right)\left(-\partial^{-2}\right)} \exp \left(-S_{i n t}(\phi)-\frac{1}{2} \int \phi\left(-\partial^{2}\right) K^{-1}\left(-\partial^{2} / \epsilon^{2}\right) \phi-\int J \phi\right)
$$

is well-defined for any $\epsilon<\infty$. As a formal limit, $\lim _{\epsilon \uparrow \infty} Z_{\epsilon}[J=0]=Z_{0}$. One can be more general, as will be apparent from the reasoning below. $J$ is a source restricted to vanish for momenta of order $\epsilon$ or larger. The Wilson rg flow is defined by demanding the invariance of $Z_{\epsilon}[J]$, for such $J$. It is easy to derive such a flow, not just for the low momentum correlation functions, but for $Z_{\epsilon}[J]$ itself-the normalization of the measure is important for this.

The idea behind what follows is simple: decreasing $\epsilon$ is equivalent to gradually integrating out modes with large momenta. The change in the free energy due to this explicit change in the cutoff is therefore of definite sign, due to the monotonicity of $K$. This is true even taking into account the normalization of the functional measure, assuming the positivity of the spectral representation of the two point function. On the other hand, by the definition of the Wilson rg, the free energy is left invariant under changes in $\epsilon$, provided the couplings in $S_{i n t} \equiv \sum g^{A}(\epsilon) \int \Phi_{A}$ are appropriately adjusted, so the change in the free energy upon just changing the couplings must be of the opposite sign as the change due to the explicit presence of the cutoff. $\left\{\Phi_{A}\right\}$ is a complete set of scalar operators, including the identity.

Define $\mathcal{F} \equiv-\ln Z_{\epsilon}[0] /$ Vol., the free energy density. All expressions with $1 /$ Vol. factors should be understood as implying an infinite volume limit. We have

$$
\begin{aligned}
0=\epsilon \frac{\mathrm{d}}{\mathrm{d} \epsilon} \mathcal{F}= & \frac{1}{\text { Vol. }}\left\langle\epsilon \frac{\partial}{\partial \epsilon} S_{\text {int }}\right\rangle \\
& +\frac{1}{\text { Vol. }}\left\langle\frac{1}{2} \int \phi \epsilon \frac{\partial}{\partial \epsilon} K^{-1}\left(-\partial^{2} / \epsilon^{2}\right)\left(-\partial^{2}\right) \phi\right\rangle-\frac{1}{2} \int K \epsilon \frac{\partial}{\partial \epsilon} K^{-1} .
\end{aligned}
$$


This amounts to

$$
\frac{1}{\text { Vol. }}\left\langle\epsilon \frac{\partial}{\partial \epsilon} S_{\text {int }}\right\rangle+\frac{1}{\text { Vol. }} \int \frac{1}{2} \epsilon \frac{\partial}{\partial \epsilon} K^{-1}\left(p^{2} / \epsilon^{2}\right) p^{2}\left(\langle\phi \phi\rangle-\frac{K\left(p^{2} / \epsilon^{2}\right)}{p^{2}}\right)=0 .
$$

I now wish to argue that the second term is positive on general grounds.

By picking $f(x)=K\left(x / \epsilon^{2}\right)^{-1}$, the spectral representation becomes

$$
\langle\phi(p) \phi(-p)\rangle=\int_{0}^{\infty} \mathrm{d} m^{2} \rho\left(m^{2}\right) \frac{K\left(p^{2} / \epsilon^{2}\right)}{p^{2}+K\left(p^{2} / \epsilon^{2}\right) m^{2}},
$$

with

$$
\left.\rho\left(m^{2}\right) \equiv(2 \pi)^{d-1}\langle 0|\phi(x=0) \delta(\hat{p}-p) \phi(x=0)| 0\rangle\right|_{p^{2}+K\left(\left|p^{2}\right| / \epsilon^{2}\right) m^{2}=0} .
$$

An implicit assumption here is the existence of 'vacuum' boundary conditions for the functional integration - due to the higher derivative form of the cutoff theory, a Hamiltonian definition is problematic.

From the spectral representation, we note

$$
\lim _{p^{2} \uparrow \infty} p^{2} K^{-1}\left(p^{2} / \epsilon^{2}\right)\langle\phi(p) \phi(-p)\rangle=\int_{0}^{\infty} \mathrm{d} m^{2} \rho\left(m^{2}\right) .
$$

In cutoff perturbation theory, with the assumed form of $K$, we therefore have

$$
\int \mathrm{d} m^{2} \rho\left(m^{2}\right)=1
$$

This is valid to all orders in perturbation theory. This normalization of the spectral measure in the cutoff theory, and the positivity of the spectral measure, are the crucial assumptions in the present discussion. The normalization assumption is not true for interacting renormalized field theories, hence the present argument does not admit any naïve generalization to the field theoretic rg.

It now follows that

$$
\frac{1}{\text { Vol. }} \int \frac{1}{2} \epsilon \frac{\partial}{\partial \epsilon} K^{-1}\left(p^{2} / \epsilon^{2}\right) p^{2}\left(\langle\phi \phi\rangle-\frac{K\left(p^{2} / \epsilon^{2}\right)}{p^{2}}\right)>0,
$$

since

$$
\frac{K\left(p^{2} / \epsilon^{2}\right)}{p^{2}} \geq \frac{K\left(p^{2} / \epsilon^{2}\right)}{p^{2}+K\left(p^{2} / \epsilon^{2}\right) m^{2}}
$$

for $m^{2} \geq 0$, and $K$ is monotone. This quantity is certainly finite to all orders in perturbation theory, since we have normalized the measure appropriately. This finally implies

$$
\frac{1}{\text { Vol. }}\left\langle\epsilon \frac{\partial}{\partial \epsilon} S_{\text {int }}\right\rangle=\sum \epsilon \frac{\partial}{\partial \epsilon} g^{A}(\epsilon) \frac{1}{\operatorname{Vol} .}\left\langle\int \Phi_{A}\right\rangle \equiv \sum \beta_{W}^{A} \partial_{A} \mathcal{F}<0
$$


This is precisely what we wished to show, since we have shown that $\mathcal{F}$ decreases along Wilson rg trajectories, defined by the vector field $\beta_{W}$, at every fixed $\epsilon$.

Some caveats: (1) If the Wilson rg flow leads to theories where the positivity assumption no longer holds, this argument breaks down. Such theories would not appear to make sense as Euclidean field theories. (2) This argument does not extend in any straightforward way to gauge theories, because the only gauge-invariant 'physical' regulator known is the lattice, where the separation of kinetic and interaction terms in the action is unnatural.

(3) This argument only holds to all orders in perturbation theory.

It may be possible to use this decrease of $\mathcal{F}$ to derive a $c$ theorem for the field theoretic rg, even though the intermediate steps cannot be translated. The necessary ingredients are (a) to control the manner in which the Wilson rg flow converges to the submanifold of relevant and marginal couplings, and (b) to control a change of variables from the couplings that occur in the cutoff action to the normalization conditions that describe this relevant/marginal coupling submanifold.

Acknowledgements: I am grateful to R.C. Myers for helpful conversations. I would also like to thank J. Cardy, D. Friedan, D. Gross and M. Lässig for useful discussions and comments. This work was supported in part by NSF Grant No. PHY90-21984.

\section{References}

1. K. Wilson, in Collective properties of physical systems, (B. Lundqvist and S. Lundqvist, eds.), Academic Press, New York, 1973

2. D.J. Wallace and R.K.P. Zia, Ann. Phys. 92 (1975) 142

3. D.J. Gross, in Field theory and critical phenomena, (R. Balian and J. Zinn-Justin, eds.), North-Holland, Amsterdam, 1975

4. A.B. Zamolodchikov, JETP Lett. 43 (1986) 730

5. E.C.G. Stueckelberg and A. Petermann, Helv. Phys. Acta 26 (1953) 499; M. GellMann and F.E. Low, Phys. Rev. 95 (1954) 1300; C.G. Callan, Phys. Rev. D2 (1970) 1541; K. Symanzik, Comm. Math. Phys. 23 (1971) 227

6. K.G. Wilson and J.B. Kogut, Phys. Rep. 12C (1974) 75; F. Wegner, in Phase transitions and critical phenomena, vol. 6, (C. Domb and M. Green, eds.), Academic Press, New York, 1976; J. Polchinski, Nucl. Phys. B231 (1984) 269

7. J. Hughes and J. Liu, Nucl. Phys. B307 (1988) 183

8. C. di Castro and G. Jona-Lasinio, in Phase transitions and critical phenomena, vol. 6, (C. Domb and M. Green, eds.), Academic Press, New York, 1976

9. J. Cardy, Phys. Lett. 215B (1988) 749 
10. A. Cappelli, D. Friedan and J. Latorre, Nucl. Phys. B352 (1991) 616

11. G. Jona-Lasinio, in Collective properties of physical systems, (B. Lundqvist and S. Lundquist, eds.), Academic Press, New York, 1973

12. P. Haagensen, Y. Kubyshin, J. Latorre and E. Moreno, 'Gradient flows from an approximation to the exact renormalization group', Barcelona preprint (1993), and references therein. 